\title{
PREDICTORS OF RETENTION IN A RANDOMISED TRIAL OF SMOKING \\ CESSATION IN LOW-SOCIOECONOMIC STATUS AUSTRALIAN SMOKERS
}

Courtney RJ, ${ }^{1 *}$ Clare P, ${ }^{1}$ Boland V, ${ }^{1}$ Martire K.A,${ }^{1,2}$ Bonevski B, ${ }^{3}$ Hall W, ${ }^{4}$ Siahpush $M,{ }^{5}$

Borland R, ${ }^{6}$ Doran $\mathrm{C},{ }^{7}$ West $\mathrm{R}^{8}$, Farrell M, ${ }^{1}$ Mattick, R.P. ${ }^{1}$

${ }^{1}$ National Drug \& Alcohol Research Centre, University of New South Wales (UNSW), Australia

${ }^{2}$ School of Psychology, UNSW, Australia ORCID: http://orcid.org/0000-0002-5324-0732

${ }^{3}$ School of Medicine \& Public Health, Faculty of Health and Medicine, University of Newcastle, Australia

${ }^{4}$ UQ Centre for Clinical Research, University of Queensland, Australia

${ }^{5}$ College of Public Health, University of Nebraska Medical Center, United States

${ }^{6}$ Centre for Behavioural Research in Cancer, Cancer Council Victoria, Australia

${ }^{7}$ School of Human, Health and Social Sciences, Central Queensland University, Brisbane, Australia

${ }^{8}$ UCL Department of Epidemiology \& Public Health, University College London, United Kingdom

* Corresponding author. Ryan J Courtney. UNSW, National Drug \& Alcohol Research Centre, R3

Building, Room G33, Sydney, NSW, 2052. e-mail: r.courtney@unsw.edu.au Tel: 0289361004 Fax: 0293850222

Keywords: smoking cessation, socioeconomic factors, vulnerable populations, method, retention

XXXX words

Australian and New Zealand Clinical Trials Registry (ACTRN12612000725864). 


\section{ABSTRACT}

Background High attrition rates can threaten the validity of smoking cessation trial findings. Little has been published on the factors associated with retention in smoking cessation trials, especially those with smokers of low-socioeconomic status (low-SES).

Objectives To examine the factors associated with retention of low-SES smokers in the Australian Financial Interventions for Smoking Cessation Among Low-Income Smokers (FISCALS) trial.

Methods 1047 low-SES smokers were randomised. Participants completed computer assisted telephone interviews (CATIs) at baseline, 2-month and 8-month follow-up. Smoking-related, health-related, behavioural, sociodemographic and recruitment sources association with retention on the primary outcome of trial retention at final 8-month follow-up were examined using binary logistic regression.

Results 946 participants (90\%) completed the 2-month follow-up interview and 880 participants (84\%) completed the final follow-up interview. Retention at 8 months was associated with higher motivation to quit (OR: 1.15 ; $95 \%$ CI: $1.04,1.27 p<.01)$, more quit attempts (OR: $1.20 ; 95 \%$ CI: 1.04, $1.40 p<.05)$, increasing age (OR: 1.05; 95\% CI: 1.03, $1.07 p<.01$ ), and higher level of education (OR: 2.24; 95\% CI: 1.45, $3.46 p<.01)$. Lower retention at 8-months occurred for those recruited from posters placed in Department of Human Service Centrelink Offices (OR: 0.56; 95\% CI: $0.35,0.89, p<.05)$ than in participants recruited from telephone Quitline or Newspaper advertisements. No significant associations were found between health-related or behavioural factors and retention.

Conclusions Rigorous retention strategies and comprehensive participant tracking can ensure high retention and minimal loss-to-follow-up in low SES smokers.

\section{INTRODUCTION}


Participant attrition ${ }^{[1]}$ is a potential problem in interpreting the findings of clinical trials especially when participants permanently drop out of a study. ${ }^{[2]}$ There are two principal types of participant attrition : (i) drop out/ withdrawal i.e. participants that no longer wish to participate in any further data collection/ study demands; and (ii) loss to follow-up i.e. participants who are not retained/ or lost without reason. ${ }^{[3]}$ It is a common stumbling problem in clinical trials. For example, a review of health care intervention randomised control trials (RCTs) in six major journals, ${ }^{[1]}$ found that $48 \%$ of trials that reported a sample size calculation failed to retain adequate numbers at outcome assessment because of loss to follow-up. ${ }^{[1,4]}$

Excessive loss to follow-up can prolong recruitment, reduce statistical power, threaten the internal validity of study findings, compromise the generalisability of study outcomes, and waste money. ${ }^{[5,6]}$ Study results can be biased when participants retained differ from those who are not ${ }^{[7]}$ and bias may be even more pronounced when loss to follow-differs up between the intervention and comparison group $(s) .{ }^{[8]}$ Assessment of the characteristics and factors associated with attrition is essential ${ }^{[3]}$ to assess for selection biases and loss of statistical power ${ }^{[3,9,10]}$ and these need to be considered in data analysis and interpretation. ${ }^{[3,11,12]}$ As a rule of thumb, loss to follow-up under $5 \%$ will result in little bias but over $20 \%$ loss can significantly threaten study validity. ${ }^{[4,8]}$ Previous studies have indicated that often those participants with incomplete follow-up data, while similar at baseline, may be systematically different at follow-up. ${ }^{[13,14]}$ Consequently this can limit the generalisability of the study and lead to incorrect inferences about treatment effects in the cohort. ${ }^{[13]}$ It is imperative that researchers get as close to complete follow-up data as possible. ${ }^{[4,8]}$

Low-socioeconomic status (low-SES) populations have lower participation rates and higher drop out or loss to follow-up rates. ${ }^{[15]}$ Low-SES populations also have characteristics that make followup more difficult, namely, elevated rates of substance abuse and mental health disorders, housing instability, intermittent telephone access and use, incarceration, and lesser exposure to research. ${ }^{[16-}$ ${ }^{18]}$ Lower education, low health literacy, and financial stress are also associated with incomplete research follow-up. ${ }^{[13]}$ 
Cochrane systematic reviews ${ }^{[19]}$ suggests that adequate retention rates can be achieved by implementing the following strategies: obtaining multiple contact details from participants, making frequent participant contact, and using incentives and participant reimbursement. However, there is a paucity of information on what strategies are effective at maximising study retention rates among low-SES populations ${ }^{[15]}$. A randomized, cross-sectional study trial comparing data collection methods identified more complete data for telephone surveys compared to mail-based surveys among disadvantaged populations with limited-English proficiency. ${ }^{[20]}$ In addition, systematic reviews indicate that larger cash amounts lead to higher study retention in disadvantaged population groups. $^{[15]}$

There is little research on optimal methods of ensuring high retention of low-SES populations in research studies, particularly among low-SES smoking populations. For example, a recent systematic review of barriers to retaining participants and strategies to maximise retention rates in health research among socially disadvantaged groups identified only 26 studies. ${ }^{[15]}$ Only three studies were conducted among socially disadvantaged smoking groups, ${ }^{[21-23]}$ and these were African American smokers and only involved participants enrolled in a RCT. ${ }^{[21]}$

In the general smoking population, smoking-related, socio-demographic, behavioural, and healthrelated factors have been linked to poor retention, but little is known about the role of these factors and the recruitment source in retention of low-SES smokers. Length of previous quit attempts ${ }^{[24,25]}$ and confidence in quitting ${ }^{[26]}$ are associated with study retention but evidence is mixed for cigarettes smoked per day ${ }^{[26-28]}$. On the whole, the association between study retention and other sociodemographic characteristics (e.g. age, ${ }^{[25,29]}$, education level, ${ }^{[24,28]}$ sex,${ }^{[30]}$ and number of dependent children $)^{[25]}$, behavioural/ psychological factors (e.g. weight concerns ${ }^{[25]}$, feelings of guilt, $\mathrm{IQ}^{[31,32]}$ ) and health-related factors (e.g. depression ${ }^{[28]}$, body mass index (BMI) and other health risk behaviours $)^{[3,33-36]}$ is conflicting . Further, there is an absence of data from smoking cessation clinical trials in socially disadvantaged populations. ${ }^{[15]}$ Many studies have failed to analyse the independent contributions of these factors to follow-up ${ }^{[13]}$. Little effort has been made to investigate 
other factors that may be more salient in low-SES groups, for example mental health disorders and poorer physical health. ${ }^{[13]}$ If factors associated with drop out in smoking cessation trials in low-SES populations are identifiable at study commencement, measures can be taken to enhance retention. ${ }^{\text {[37] }}$

\section{Aims and objectives}

The aims of this study were (1) to describe the follow-up retention rates achieved in the Financial Interventions for Smoking Cessation Among Low-Income Smokers (FISCALS) RCT and (2) to identify whether smoking-related, health-related, behavioural, socio-demographic characteristics or recruitment source were associated with participant retention at 2- or 8-month follow-ups.

\section{METHOD}

\section{Study design}

The FISCALs pragmatic RCT was funded (APP1021862) by the Australian National Health and Medical Research Council (NHMRC) and the study protocol is published elsewhere. ${ }^{[38]}$ The design of this trial was in accordance with the Consolidated Standards of Reporting Trials (CONSORT) statement and the trial was registered with the Australian and New Zealand Clinical Trials Registry (ACTRN12612000725864). Details of the FISCALs trial and study measures are described elsewhere. ${ }^{[38]}$ For the purposes of this study participant recruitment, retention rates and retention strategies adopted will be explained in more detail. 


\section{Participants}

The Consolidated Standards of Reporting Trials (CONSORT) diagram for the FISCALs trial is presented in Supplementary Material 1. Participants were eligible for the FISCALs trial if they were: aged 18 years or over; currently smoking at least 10 cigarettes per day; currently in receipt of a government pension or allowance (proxy for low-SES); motivated to quit; willing to make a quit attempt in the next month; not currently taking any smoking cessation medications; willing to receive telephone-based support to help quit smoking; willing to comply with study measurement demands; able to read and understand English language; and have a home or mobile telephone. As part of this trial all eligible participants were mailed an 8-week supply of combination NRT comprising the $21 \mathrm{mg} / 24$-hour nicotine patch plus either $2 \mathrm{mg}$ gum or lozenge.

\section{Recruitment}

Participants were recruited via three recruitment sources: 1 . Telephone Quitline services; 2. Poster advertisements in Department of Human Services Centrelink Customer Service Centres (DHS CSC); and 3. Newspaper advertisements. Multiple recruitment strategies as outlined in detail below were adopted to ensure high reach to low-SES smokers. In recruitment source 1 (Quitline) participants were offered trial participation from their Quitline telephone support counsellor and the research team made contact with those willing to hear more about the study. In recruitment source 2 (Centrelink posters) and 3 (newspaper advertisements) recruitment was reactive and participants had to self-refer and make direct contact with the research team (See Supplementary Files 2 and 3). A detailed overview of the recruitment methodology for each recruitment channel adopted can be found elsewhere in the study protocol paper. ${ }^{[38]}$

\section{Computer-assisted telephone interviews (CATIs)}

Low-SES smokers were recruited from April, 2013 until September, 2014. ${ }^{[38]}$ Participants completed three computer assisted telephone interviews (CATI), at baseline, two and eight months. 
Interviews were conducted by an independent survey firm. Participants were reimbursed $\$ 40$ for completing each interview.

\section{CATI follow-up protocol}

Participants were contacted by telephone to complete baseline and follow-up interviews. The HVRF call centre operated from 9am until 8.30pm Monday to Friday from April 2013 until 26 January 2014 and Monday to Thursday from 27 January 2014 until completion of data collection in September 2014. Participants were offered interviews on Saturdays in exceptional circumstances. Interviewers attempted to make contact using participants' preferred contact number via either fixed home line or mobile phone. Prior to each interview, participants gave their preferred time to be contacted and were allocated to a convenient timeslot. If participants could not be contacted, a voicemail message was left asking them to call back as early as possible. If participants' telephone line was no longer in use, the research team would follow-up with any secondary contact that participants provided.

\section{Retention strategies}

Participant retention strategies at both 2- and 8-month follow-up included: (i) scheduling interviews with the participant (no cold calling); (ii) recording multiple contact details i.e. land/ mobile telephone numbers/ email address; (iii) recording secondary contact details; (iv) $\$ 40$ reimbursements for each interview; (v) multiple call attempts (up to 12 calls); and (vi) sending multiple reminders including letters/text-messages/emails. Letters were sent to participants before their 2- and 8-month follow-up interview alongside a study fridge magnet and study business card with the scheduled interview date and toll-free study number listed. In addition, a text message was sent one week prior to the scheduled interview to remind participants of the interview and to encourage them to update the research team if their contact details were to change. Emails were also sent where possible with a prompt to update contact details. If participants did not complete 
their interview after the seventh call attempt, the research team sent: (i) an additional reminder letter; (ii) email reminder, and (iii) contacted the participants' secondary contact if provided.

\section{Terminology and definitions}

Participants were deemed to have withdrawn from the study if they returned their "revocation of consent" form or contacted the research team by telephone or email and requested no further contact. Any voluntarily provided reason(s) for withdrawal were recorded by the research team. Participants were considered lost to follow-up if they had not completed their final follow-up despite several call attempts, did not answer any call attempts or their next of kin had advised the research team that they had died.

\section{Predictors of retention}

We included self-reported items previously associated with retention in longitudinal and clinical trials and variables that the research team hypothesised may be associated with retention. The selfreported data collected via CATI included: smoking-related, socio-demographics, health-related, and general psychological factors (see Supplementary Material 4). Most of these data were collected at the baseline CATI. The exception was self-reported "quit status" and intention to quit which were collected at the 2-month follow-up interview. Details of all included data can be seen in Supplementary Material 4.

\section{Smoking-related data}

We collected data on heaviness of smoking index, ${ }^{[39]}$ number of recent quit attempts, urges to smoke, self-efficacy/ confidence in quitting, and motivation to quit. In predicting final retention at 8-month follow-up, quit status at 2-month follow-up was also used with participants coded into one of three levels: 1) quit (defined by self-reported 7-day abstinence); 2) not quit but plan to give up in the next six months; or 3) not quit and do not plan to quit in the next 6 months.

\section{Health-related data}


We collected the following measures of substance use/addiction: Alcohol use (AUDIT-C), ${ }^{[40]}$ use of cannabis in last 12 months, recent drug or alcohol treatment, and problem gambling. ${ }^{[41]}$

Mental/Physical health data included: previously diagnosed or treated for a mental health condition, psychological wellbeing (DASS-21),${ }^{[42]}$ and health state measured via the EQ Visual Analogue Scale (EQ VAS) scale from the Euro-Qol 5D (EQ-5D). ${ }^{[43]}$

\section{General psychological constructs}

General psychological constructs included financial stress, ${ }^{[44-47]}$ literacy as measured by the Test of Premorbid Function (TOPF) ${ }^{[48]}$ numeracy through the WAIS-IV arithmetic subscale, ${ }^{[49]}$ and impulsiveness measured using the Barratt Impulsiveness Scale. ${ }^{[50]}$

\section{Socio-demographic data}

These included: sex, age, Indigenous status, level of education, employment status, marital status, number of children in household, proportion of social network who smoke, and a measure of social disadvantage, the Socio-Economic Index for Areas (SEIFA). ${ }^{[51]}$

\section{Statistical analysis}

Binary logistic regression models were used to assess predictors of retention at 2-months and 8months. Covariates included were the same across models, with the exception of quit status, which was obtained in the 2-month interview and thus could only be used in the model of 8-month retention. For comparison purposes, bivariate analyses were also conducted for all variables. All analyses were conducted in Stata 13.1. ${ }^{[52]}$

\section{RESULTS}

\section{Sample}

In total 2,557 people were assessed for eligibility, of which 1,047 (41\%) participants were randomised. the largest group who were not randomised were deemed ineligible because not of 
low-SES $(\mathrm{n}=555,22 \%)$. Of those randomised, 946 (90\%) completed the 2-month follow-up and 880 (84\%) completed the final 8-month follow-up. Further details of recruitment and retention can be seen in the CONSORT diagram (see Supplementary Figure 1). Retention rate at 8-month follow-up were similar for the control (86\%) and intervention (82\%) groups and data were combined for both groups for analysis.

Details of the sample including differences in demographic characteristic based on retention at 2and 8-months can be seen in Table 1.

Table 1 Socio-demographic characteristics of sample by retention status

\begin{tabular}{|c|c|c|c|c|}
\hline Variable & & $\begin{array}{c}\text { Total } \\
\mathrm{n}=1,047\end{array}$ & $\begin{array}{c}\text { Retained at 2- } \\
\text { months } \\
n=946\end{array}$ & $\begin{array}{c}\text { Retained at 8- } \\
\text { months } \\
n=880\end{array}$ \\
\hline \multirow{3}{*}{ Sex } & Female & $53.2 \%$ & $52.7 \%$ & $52.7 \%$ \\
\hline & Male & $46.6 \%$ & $47.0 \%$ & $47.0 \%$ \\
\hline & Other & $0.2 \%$ & $0.2 \%$ & $0.2 \%$ \\
\hline \multirow{6}{*}{ Age } & $18-24$ & $8.3 \%$ & $7.7 \%$ & $6.7 \%$ \\
\hline & $25-34$ & $16.1 \%$ & $15.5 \%$ & $14.7 \%$ \\
\hline & $35-44$ & $22.2 \%$ & $21.8 \%$ & $21.4 \%$ \\
\hline & $45-54$ & $24.5 \%$ & $25.1 \%$ & $25.8 \%$ \\
\hline & $55-64$ & $18.0 \%$ & $18.6 \%$ & $19.3 \%$ \\
\hline & $65+$ & $10.8 \%$ & $11.2 \%$ & $12.2 \%$ \\
\hline \multirow{2}{*}{ ATSI Status } & No & $93.3 \%$ & $93.1 \%$ & $93.7 \%$ \\
\hline & Yes & $6.7 \%$ & $6.9 \%$ & $6.3 \%$ \\
\hline \multirow{2}{*}{ Level of education } & High school or lower & $63.4 \%$ & $62.6 \%$ & $60.6 \%$ \\
\hline & More than high school & $36.6 \%$ & $37.4 \%$ & $39.4 \%$ \\
\hline \multirow{3}{*}{ Employment status } & Employed & $15.3 \%$ & $14.8 \%$ & $15.4 \%$ \\
\hline & Unemployed - in workforce & $26.3 \%$ & $26.7 \%$ & $25.1 \%$ \\
\hline & $\begin{array}{l}\text { Unemployed - not in } \\
\text { workforce }\end{array}$ & $58.4 \%$ & $58.5 \%$ & $59.5 \%$ \\
\hline \multirow{2}{*}{ Marital status } & Married/partnered/de facto & $30.6 \%$ & $31.0 \%$ & $30.6 \%$ \\
\hline & Separated/divorced/widowed & $35.0 \%$ & $34.8 \%$ & $35.7 \%$ \\
\hline
\end{tabular}




\begin{tabular}{lllll} 
& Single/never married & $34.4 \%$ & $34.2 \%$ & $33.7 \%$ \\
\hline & None & $13.8 \%$ & $14.0 \%$ & $14.2 \%$ \\
\multirow{2}{*}{$\begin{array}{l}\text { Proportion of smokers } \\
\text { in social network }\end{array}$} & A few & $28.5 \%$ & $28.6 \%$ & $29.7 \%$ \\
& Half & $16.5 \%$ & $16.7 \%$ & $16.5 \%$ \\
& Most & $25.4 \%$ & $24.5 \%$ & $24.3 \%$ \\
\multirow{2}{*}{ SEIFA } & All & $15.9 \%$ & $16.2 \%$ & $15.3 \%$ \\
\hline
\end{tabular}

\section{Contact attempts}

All participants were contacted repeatedly over a period of two to four-weeks from the date of any scheduled interview. The average number of calls to participants who completed interviews was 2.1 for baseline, 3.1 for 2-month follow-up and 3.5 for 8-month follow-up. Those who failed to complete an interview received an average of 10.0, 9.1 and 9.0 calls respectively. All participants who had not completed the interview after 7 calls were also sent reminder letters, emails and/or SMS messages, and were reminded that they would be reimbursed $\$ 40$ for completion. Of the 1047 randomised participants 59\% (616/1047) provided secondary contact details.

\section{Factors associated with retention}

\section{Smoking-related}

As seen in Table 2, motivation to quit was significantly associated with both 2-month and 8-month retention, both when unadjusted and adjusting for other covariates; higher motivation was associated with increased odds of completing both the 2-months (OR: 1.16; 95\% CI: 1.03, 1.30, $p$ $<.05)$ and 8-months interviews (OR: 1.15; 95\% CI: 1.04, 1.27, $p<.01)$. Unadjusted associations were similar, as seen in Table 3. After adjustment, the number of recent quit attempts was associated with retention at 8-months (OR: 1.20; 95\% CI: 1.04, 1.40, $p<.05$ ) but not at 2-months. No other smoking-related variables were associated with retention. Notably, we did not find a difference in retention at 8-months based on participants' self-reported quit status at 2-months. 
Table 2 Adjusted associations between retention and a range of covariates

\begin{tabular}{|c|c|c|c|c|c|}
\hline \multirow{2}{*}{\multicolumn{2}{|c|}{ Variable }} & \multicolumn{2}{|c|}{ 2-month retention } & \multicolumn{2}{|c|}{ 8-month retention } \\
\hline & & OR $(95 \% \mathrm{CI})$ & p-value & OR $(95 \%$ CI $)$ & p-value \\
\hline \multicolumn{6}{|c|}{ Smoking related variables } \\
\hline \multicolumn{2}{|c|}{ Heaviness of smoking index at baseline } & $0.93(0.77,1.12)$ & $\mathrm{p}=0.431$ & $0.89(0.76,1.05)$ & $\mathrm{p}=0.172$ \\
\hline \multirow{3}{*}{$\begin{array}{l}\text { Quit status at 2- } \\
\text { month interview }\end{array}$} & Quit & & & 1.00 & \\
\hline & $\begin{array}{l}\text { Not quit - plan to quit in next } \\
6 \text { months }\end{array}$ & & & $1.04(0.83,1.30)$ & $\mathrm{p}=0.739$ \\
\hline & $\begin{array}{l}\text { Not quit - no plan to quit in } \\
\text { next } 6 \text { months }\end{array}$ & & & $0.93(0.78,1.12)$ & $\mathrm{p}=0.449$ \\
\hline \multicolumn{2}{|c|}{ Number of recent quit attempts } & $1.11(0.94,1.30)$ & $\mathrm{p}=0.217$ & $1.20(1.04,1.40)$ & $\mathrm{p}=0.014$ \\
\hline \multicolumn{2}{|c|}{ Urges to smoke } & $0.85(0.63,1.15)$ & $\mathrm{p}=0.295$ & $1.04(0.83,1.30)$ & $\mathrm{p}=0.739$ \\
\hline \multicolumn{2}{|c|}{ Self-efficacy/confidence in quitting } & $0.94(0.76,1.16)$ & $\mathrm{p}=0.566$ & $0.93(0.78,1.12)$ & $\mathrm{p}=0.449$ \\
\hline \multicolumn{2}{|c|}{ Motivation to quit } & $1.16(1.03,1.30)$ & $\mathrm{p}=0.014$ & $1.15(1.04,1.27)$ & $\mathrm{p}=0.007$ \\
\hline \multicolumn{6}{|c|}{ Substance use/addiction } \\
\hline \multicolumn{2}{|c|}{ AUDIT-C Score } & $1.07(1.00,1.16)$ & $\mathrm{p}=0.065$ & $1.00(0.94,1.06)$ & $\mathrm{p}=0.916$ \\
\hline \multirow{2}{*}{ Use of cannabis } & No & 1.00 & & 1.00 & \\
\hline & Yes & $0.95(0.53,1.73)$ & $\mathrm{p}=0.878$ & $1.24(0.76,2.02)$ & $\mathrm{p}=0.396$ \\
\hline \multirow{2}{*}{$\begin{array}{l}\text { Recent drug } \\
\text { treatment }\end{array}$} & No & 1.00 & & 1.00 & \\
\hline & Yes & $1.07(0.55,2.08)$ & $\mathrm{p}=0.837$ & $0.75(0.46,1.23)$ & $\mathrm{p}=0.258$ \\
\hline \multirow{2}{*}{ Problem gambling } & No & 1.00 & & 1.00 & \\
\hline & Yes & $1.20(0.75,1.93)$ & $\mathrm{p}=0.455$ & $0.95(0.64,1.42)$ & $\mathrm{p}=0.803$ \\
\hline \multicolumn{6}{|c|}{ Mental/Physical Health } \\
\hline \multirow{6}{*}{$\begin{array}{l}\text { Diagnosed/treated for } \\
\text { mental health } \\
\text { condition } \\
\text { DASS - Depression } \\
\text { DASS - Anxiety } \\
\text { DASS - Stress } \\
\text { EQ VAS }\end{array}$} & No & 1.00 & & 1.00 & \\
\hline & Yes & $1.26(0.77,2.06)$ & $\mathrm{p}=0.353$ & $1.18(0.78,1.77)$ & $\mathrm{p}=0.435$ \\
\hline & & $1.02(0.99,1.05)$ & $\mathrm{p}=0.217$ & $1.02(0.99,1.04)$ & $\mathrm{p}=0.217$ \\
\hline & & $1.02(0.98,1.05)$ & $\mathrm{p}=0.339$ & $1.00(0.97,1.03)$ & $\mathrm{p}=0.876$ \\
\hline & & $0.96(0.93,1.00)$ & $\mathrm{p}=0.035$ & $0.98(0.96,1.01)$ & $\mathrm{p}=0.266$ \\
\hline & & $1.00(0.99,1.01)$ & $\mathrm{p}=0.907$ & $1.00(0.99,1.01)$ & $\mathrm{p}=0.794$ \\
\hline \multicolumn{6}{|c|}{ General Psychological Constructs } \\
\hline \multicolumn{2}{|c|}{ Financial Stress Scale } & $1.12(0.97,1.29)$ & $\mathrm{p}=0.129$ & $0.96(0.85,1.08)$ & $\mathrm{p}=0.472$ \\
\hline \multicolumn{2}{|l|}{ Literacy } & $1.00(0.98,1.03)$ & $\mathrm{p}=0.907$ & $1.00(0.98,1.02)$ & $\mathrm{p}=0.909$ \\
\hline \multicolumn{2}{|l|}{ Numeracy } & $1.02(0.92,1.14)$ & $\mathrm{p}=0.649$ & $1.00(0.92,1.09)$ & $\mathrm{p}=0.933$ \\
\hline Impulsiveness - Moto & & $0.99(0.92,1.06)$ & $\mathrm{p}=0.691$ & $1.01(0.96,1.07)$ & $\mathrm{p}=0.609$ \\
\hline Impulsiveness - Non- & anning & $0.93(0.87,1.00)$ & $\mathrm{p}=0.047$ & $0.96(0.90,1.02)$ & $\mathrm{p}=0.156$ \\
\hline Impulsiveness - Atten & onal & $1.07(0.99,1.16)$ & $\mathrm{p}=0.073$ & $1.01(0.95,1.07)$ & $\mathrm{p}=0.814$ \\
\hline Socio-demographic & riables & & & & \\
\hline & Female & 1.00 & & 1.00 & \\
\hline Sex & Male & $1.12(0.70,1.80)$ & $\mathrm{p}=0.626$ & $1.20(0.81,1.79)$ & $\mathrm{p}=0.357$ \\
\hline & Other & & & & \\
\hline Age & & $1.04(1.02,1.06)$ & $\mathrm{p}=0.001$ & $1.05(1.03,1.07)$ & $\mathrm{p}<0.001$ \\
\hline & No & 1.00 & & 1.00 & \\
\hline A1S1 Status & Yes & $1.60(0.60,4.27)$ & $\mathrm{p}=0.352$ & $0.82(0.42,1.58)$ & $\mathrm{p}=0.548$ \\
\hline I avel of education & High school or lower & 1.00 & & 1.00 & \\
\hline Level of education & More than high school & $1.46(0.89,2.39)$ & $\mathrm{p}=0.135$ & $2.24(1.45,3.46)$ & $\mathrm{p}<0.001$ \\
\hline & Employed & 1.00 & & 1.00 & \\
\hline Emplovment status & Unemployed - in workforce & $1.69(0.85,3.35)$ & $\mathrm{p}=0.132$ & $0.77(0.44,1.36)$ & $\mathrm{p}=0.370$ \\
\hline Emppoyment status & $\begin{array}{l}\text { Unemployed - not in } \\
\text { workforce }\end{array}$ & $1.14(0.62,2.10)$ & $\mathrm{p}=0.671$ & $0.85(0.49,1.48)$ & $\mathrm{p}=0.567$ \\
\hline & Married/partnered/de facto & 1.00 & & 1.00 & \\
\hline Marital status & Separated/divorced/widowed & $0.49(0.27,0.88)$ & $\mathrm{p}=0.018$ & $0.66(0.40,1.09)$ & $\mathrm{p}=0.103$ \\
\hline & Single/never married & $0.86(0.48,1.53)$ & $\mathrm{p}=0.609$ & $1.18(0.74,1.86)$ & $\mathrm{p}=0.488$ \\
\hline Number of children in & household & $0.94(0.79,1.12)$ & $\mathrm{p}=0.467$ & $0.93(0.80,1.07)$ & $\mathrm{p}=0.317$ \\
\hline Proportion social nety & rk who smoke & $0.98(0.82,1.17)$ & $\mathrm{p}=0.847$ & $0.98(0.84,1.13)$ & $\mathrm{p}=0.751$ \\
\hline
\end{tabular}




\begin{tabular}{|c|c|c|c|c|c|}
\hline SEIFA quintile & $\begin{array}{l}\text { Bottom half of SEIFA } \\
\text { Top half of SEIFA }\end{array}$ & $\begin{array}{l}1.00 \\
0.76(0.48,1.19)\end{array}$ & $\mathrm{p}=0.223$ & $\begin{array}{l}1.00 \\
0.84(0.58,1.22)\end{array}$ & $\mathrm{p}=0.354$ \\
\hline \multicolumn{6}{|l|}{ Recruitment } \\
\hline \multirow{4}{*}{ Recruitment source } & Quitline & 1.00 & & 1.00 & \\
\hline & Centrelink & $0.37(0.21,0.67)$ & $\mathrm{p}=0.001$ & $0.56(0.35,0.89)$ & $\mathrm{p}=0.015$ \\
\hline & Newspaper ads & $0.65(0.35,1.20)$ & $\mathrm{p}=0.166$ & $0.98(0.60,1.63)$ & $\mathrm{p}=0.952$ \\
\hline & Word of mouth/other & $1.08(0.48,2.41)$ & $\mathrm{p}=0.858$ & $1.46(0.78,2.73)$ & $\mathrm{p}=0.234$ \\
\hline
\end{tabular}

Table 3 Unadjusted associations between retention and a range of covariates

\begin{tabular}{|c|c|c|c|c|c|}
\hline \multirow{2}{*}{\multicolumn{2}{|c|}{ Variable }} & \multicolumn{2}{|c|}{ 2-month retention } & \multicolumn{2}{|c|}{ 8-month retention } \\
\hline & & OR $(95 \% \mathrm{CI})$ & p-value & OR $(95 \% \mathrm{CI})$ & p-value \\
\hline \multicolumn{6}{|c|}{ Smoking related variables } \\
\hline \multicolumn{2}{|c|}{ Heaviness of smoking index at baseline } & $0.99(0.85,1.17)$ & $\mathrm{p}=0.936$ & $0.94(0.83,1.08)$ & $\mathrm{p}=0.392$ \\
\hline \multirow{3}{*}{$\begin{array}{l}\text { Quit status at 2- } \\
\text { month interview }\end{array}$} & Quit & & & 1.00 & \\
\hline & $\begin{array}{l}\text { Not quit - plan to quit in } \\
\text { next } 6 \text { months }\end{array}$ & & & $1.03(0.84,1.26)$ & $\mathrm{p}=0.798$ \\
\hline & $\begin{array}{l}\text { Not quit - no plan to quit in } \\
\text { next } 6 \text { months }\end{array}$ & & & $0.99(0.85,1.16)$ & $\mathrm{p}=0.934$ \\
\hline \multicolumn{2}{|c|}{ Number of recent quit attempts } & $1.12(0.96,1.30)$ & $\mathrm{p}=0.148$ & $1.19(1.04,1.36)$ & $\mathrm{p}=0.013$ \\
\hline \multicolumn{2}{|c|}{ Urges to smoke } & $0.90(0.68,1.19)$ & $\mathrm{p}=0.455$ & $1.03(0.84,1.26)$ & $\mathrm{p}=0.798$ \\
\hline \multicolumn{2}{|c|}{ Self-efficacy/confidence in quitting } & $0.99(0.82,1.19)$ & $\mathrm{p}=0.923$ & $0.99(0.85,1.16)$ & $\mathrm{p}=0.934$ \\
\hline \multicolumn{2}{|c|}{ Motivation to quit } & $1.11(1.00,1.23)$ & $\mathrm{p}=0.048$ & $1.09(1.00,1.19)$ & $\mathrm{p}=0.039$ \\
\hline \multicolumn{6}{|c|}{ Substance use/addiction } \\
\hline \multicolumn{2}{|c|}{ AUDIT-C Score } & $1.05(0.98,1.12)$ & $\mathrm{p}=0.133$ & $0.99(0.94,1.04)$ & $\mathrm{p}=0.584$ \\
\hline \multirow{2}{*}{ Use of cannabis } & No & 1.00 & & 1.00 & \\
\hline & Yes & $0.92(0.54,1.55)$ & $\mathrm{p}=0.752$ & $0.85(0.56,1.29)$ & $\mathrm{p}=0.440$ \\
\hline \multirow{3}{*}{$\begin{array}{l}\text { Recent drug } \\
\text { treatment }\end{array}$} & No & 1.00 & & 1.00 & \\
\hline & Yes & $0.95(0.52,1.72)$ & $\mathrm{p}=0.855$ & $0.56(0.36,0.86)$ & $\mathrm{p}=0.008$ \\
\hline & No & 1.00 & & 1.00 & \\
\hline Problem gambling & Yes & $1.28(0.84,1.96)$ & $\mathrm{p}=0.253$ & $1.16(0.82,1.64)$ & $\mathrm{p}=0.403$ \\
\hline \multicolumn{6}{|c|}{ Mental/Physical Health } \\
\hline \multirow{3}{*}{$\begin{array}{l}\text { Diagnosed/treated } \\
\text { for mental health } \\
\text { condition } \\
\text { DASS - Depression }\end{array}$} & No & 1.00 & & 1.00 & \\
\hline & Yes & $1.09(0.72,1.64)$ & $\mathrm{p}=0.688$ & $0.91(0.65,1.27)$ & $\mathrm{p}=0.586$ \\
\hline & & $1.01(0.99,1.02)$ & $\mathrm{p}=0.547$ & $1.00(0.99,1.01)$ & $\mathrm{p}=0.866$ \\
\hline \multicolumn{2}{|l|}{ DASS - Anxiety } & $1.00(0.98,1.02)$ & $\mathrm{p}=0.960$ & $0.99(0.98,1.01)$ & $\mathrm{p}=0.264$ \\
\hline \multicolumn{2}{|l|}{ DASS - Stress } & $0.99(0.98,1.01)$ & $\mathrm{p}=0.366$ & $0.99(0.98,1.00)$ & $\mathrm{p}=0.146$ \\
\hline \multicolumn{2}{|c|}{ EQ VAS } & $1.00(0.99,1.01)$ & $\mathrm{p}=0.734$ & $1.00(1.00,1.01)$ & $\mathrm{p}=0.233$ \\
\hline \multicolumn{6}{|c|}{ General Psychological Constructs } \\
\hline \multicolumn{2}{|c|}{ Financial Stress Scale } & $1.00(0.89,1.12)$ & $\mathrm{p}=0.981$ & $0.86(0.79,0.95)$ & $\mathrm{p}=0.002$ \\
\hline \multicolumn{2}{|c|}{ Literacy } & $1.01(0.99,1.03)$ & $\mathrm{p}=0.431$ & $1.01(1.00,1.03)$ & $\mathrm{p}=0.098$ \\
\hline \multicolumn{2}{|c|}{ Numeracy } & $1.04(0.96,1.12)$ & $\mathrm{p}=0.367$ & $1.06(1.00,1.13)$ & $\mathrm{p}=0.068$ \\
\hline \multicolumn{2}{|c|}{ Impulsiveness - Motor } & $0.98(0.93,1.03)$ & $\mathrm{p}=0.437$ & $0.96(0.92,1.01)$ & $\mathrm{p}=0.087$ \\
\hline \multicolumn{2}{|c|}{ Impulsiveness - Non-planning } & $0.94(0.89,1.00)$ & $\mathrm{p}=0.053$ & $0.94(0.89,0.98)$ & $\mathrm{p}=0.006$ \\
\hline Impulsiveness - Att & tional & $1.02(0.96,1.08)$ & $\mathrm{p}=0.483$ & $0.96(0.92,1.00)$ & $\mathrm{p}=0.067$ \\
\hline Socio-demographi & ariables & & & & \\
\hline & Female & 1.00 & & 1.00 & \\
\hline Sex & Male & $1.20(0.79,1.82)$ & $\mathrm{p}=0.383$ & $1.12(0.80,1.56)$ & $\mathrm{p}=0.500$ \\
\hline & Other & & & & \\
\hline Age & & $1.03(1.01,1.04)$ & $\mathrm{p}=0.001$ & $1.04(1.03,1.06)$ & $\mathrm{p}<0.001$ \\
\hline & No & 1.00 & & 1.00 & \\
\hline ATSI Status & Yes & $1.42(0.56,3.62)$ & $\mathrm{p}=0.460$ & $0.67(0.37,1.21)$ & $\mathrm{p}=0.184$ \\
\hline
\end{tabular}




\begin{tabular}{|c|c|c|c|c|c|}
\hline \multirow{2}{*}{ Level of education } & High school or lower & \multicolumn{2}{|l|}{1.00} & \multicolumn{2}{|l|}{1.00} \\
\hline & More than high school & $1.41(0.90,2.21)$ & $\mathrm{p}=0.131$ & $2.28(1.54,3.37)$ & $\mathrm{p}<0.001$ \\
\hline \multirow{3}{*}{ Employment status } & Employed & 1.00 & & 1.00 & \\
\hline & Unemployed - in workforce & $1.56(0.83,2.95)$ & $\mathrm{p}=0.166$ & $0.74(0.44,1.24)$ & $\mathrm{p}=0.257$ \\
\hline & $\begin{array}{l}\text { Unemployed - not in } \\
\text { workforce }\end{array}$ & $1.36(0.79,2.33)$ & $\mathrm{p}=0.267$ & $1.11(0.69,1.80)$ & $\mathrm{p}=0.667$ \\
\hline \multirow{3}{*}{ Marital status } & Married/partnered/de facto & 1.00 & & 1.00 & \\
\hline & Separated/divorced/widowed & $0.82(0.49,1.38)$ & $\mathrm{p}=0.451$ & $1.14(0.75,1.74)$ & $\mathrm{p}=0.531$ \\
\hline & Single/never married & $0.80(0.48,1.35)$ & $\mathrm{p}=0.411$ & $0.88(0.59,1.31)$ & $\mathrm{p}=0.520$ \\
\hline \multicolumn{2}{|c|}{ Number of children in household } & $0.86(0.74,0.99)$ & $\mathrm{p}=0.040$ & $0.84(0.75,0.95)$ & $\mathrm{p}=0.006$ \\
\hline \multicolumn{2}{|c|}{ Proportion of smokers in social network } & $0.95(0.81,1.11)$ & $\mathrm{p}=0.532$ & $0.86(0.75,0.97)$ & $\mathrm{p}=0.017$ \\
\hline \multirow{2}{*}{ SEIFA quintile } & Bottom half of SEIFA & 1.00 & & 1.00 & \\
\hline & Top half of SEIFA & $0.80(0.53,1.21)$ & $\mathrm{p}=0.292$ & $0.87(0.62,1.22)$ & $\mathrm{p}=0.422$ \\
\hline \multicolumn{6}{|l|}{ Recruitment } \\
\hline \multirow{4}{*}{ Recruitment source } & Quitline & 1.00 & & 1.00 & \\
\hline & Centrelink & $0.40(0.24,0.69)$ & $\mathrm{p}=0.001$ & $0.50(0.33,0.76)$ & $\mathrm{p}=0.001$ \\
\hline & Newspaper ads & $0.80(0.45,1.41)$ & $\mathrm{p}=0.441$ & $1.16(0.74,1.82)$ & $\mathrm{p}=0.511$ \\
\hline & Word of mouth/other & $0.99(0.46,2.13)$ & $\mathrm{p}=0.980$ & $1.12(0.63,2.00)$ & $\mathrm{p}=0.707$ \\
\hline
\end{tabular}

Health-related (substance use/addiction or mental/physical health)

There was a significant association between the DASS stress subscale score and 2-month interview completion (OR: 0.96 ; 95\% CI 0.93, 1.00, $p<.05$ ): those with lower stress scores were less likely to complete the 2-month interview. No other health-related variables were associated with retention at either 2- or 8-months.

\section{General psychological constructs}

The non-planning sub-scale of the Barratt Impulsiveness scale was significantly associated with retention, with those scoring lower significantly less likely to complete the 2-month interview (OR: 0.93; $95 \%$ CI $0.87,1.00, p<.05)$, but not at 8 -months. Other psychological constructs were not associated with retention at either 2- or 8-months.

\section{Socio-demographics}

Retention was related to age, with older participants significantly more likely to complete both the 2-month (OR: 1.04; 95\% CI: 1.02, 1.06, $p<.01)$ and the 8-month interviews (OR: 1.05; 95\% CI: $1.03,1.07, p<.01)$. Retention was also related to education and marital status, as seen in Table 2: those with more than a school level of education (OR: 2.24; 95\% CI: $1.45,3.46, p<.01)$ were 
significantly more likely to complete the 8-month interview and those wo were separated, divorced or widowed (OR: $0.49 ; 95 \%$ CI: $0.27,0.88, p<.05)$ were less likely to complete the 2 -month interview than those who were single or never married.

\section{Recruitment source}

There was a strong association between recruitment source and retention, with those recruited via Centrelink being much less likely to complete either the 2-month interview (84\%; OR: 0.37 ; $95 \%$ CI: $0.21,0.67, p<.01)$ or 8 -month interview (75\%; OR: 0.56 ; 95\% CI: $0.35,0.89, p<.05$ ) compared to Quitline (93\% and $86 \%$ respectively).

\section{DISCUSSION}

To our knowledge this is the first Australian smoking cessation clinical trial for low-SES smokers to achieve over $80 \%$ retention at final follow-up . It demonstrates that high retention $(>80 \%$ at 8 month follow-up) can be achieved, in a smoking cessation RCT in low SES smokers using telephone survey methodologies . The study results also suggested that retention in this low-SES populations was less associated with general and mental health issues than might be expected in that we found that there were no significant differences in retention for those with co-morbid mental health conditions, smokers receiving additional drug or alcohol treatment, or those smokers with poorer physical health or mental wellbeing. Retention appears to be linked more with engagement and commitment to smoking cessation. In this case, those more motivated to quit and committed 
and engaged in trying to quit (marked by number of recent quit attempts) were more likely to remain in the study.

We did find that other socio-demographic characteristics were linked to retention, namely, increasing age ${ }^{[25,29]}$ and higher level of education ${ }^{[24]}$ that have been previously associated with higher rates of retention in smoking cessation trials. It is worth noting that recruitment source was also significantly associated with retention, with those recruited through Centrelink less likely to complete the follow-up interviews. This suggests that serious consideration must be given to the methods used to recruit participants from disadvantaged populations, if high rates of retention are to be maintained.

The retention rate at 8 -month follow-up (84\%) in this study compares very favourably to the only other published Australian smoking cessation trials in socially disadvantaged populations. ${ }^{[53]}$ Few behavioural smoking cessation clinical trials have been undertaken internationally among socially disadvantaged groups ${ }^{[54]}$ and only one of 32 published studies in a recent systematic review ${ }^{[55]}$ was conducted in Australia among smokers with a psychotic disorder. It achieved a retention rate of $83 \%(247 / 298)$ at 12-month final follow-up, ${ }^{[53]}$ but factors associated with retention were not investigated One recent RCT published in 2014 among 430 homeless smokers from US emergency shelters and transitional housing units identified a overall retention rate of $75 \%$ at 26 -week followup, and found similar to the current study found that age was associated with increased retention, and that alcohol, other drug use and health-related constructs were not associated with retention. ${ }^{[37]}$

Motivation to quit (at 2-month and 8-month follow-up) and number of previous quit attempts were associated with retention in the current study, although the association with number of previous quit attempts did not persist at 8-month follow-up. This is not entirely consistent with the wider literature, as some studies have identified an association between increased motivation and confidence to quit and retention, ${ }^{[26,57,58]}$ while others have found the opposite association or no association at all. ${ }^{[37]}$ It is noteworthy that a number of the more commonly known 
sociodemographic characteristics that have been associated with retention in other smoking cessation studies, ${ }^{[15,21,59]}$ were not associated with retention in our study, e.g. sex, marital status, nicotine dependence, and self-reported quit status at 2-month follow-up . The heterogeneity in retention research, including differing study designs, populations investigated, in combination with varying characteristics explored makes it difficult to extrapolate a clear association. Consequently, further research is required to resolve this issue, particularly among socially disadvantaged population groups. ${ }^{[37]}$

Methods of recruitment have been related to participant retention.$^{[7,60]}$ Participants recruited via posters located in Department of Human Services, Centrelink Customers Service locations were significantly less likely to be retained compared to other recruitment sources. The Centrelink recruitment strategy was reactive. A recent study found that retention was five times greater for low-income pregnant participants enrolled in a second-hand smoke reduction trial when recruited both reactively and proactively compared to reactively alone. ${ }^{[61]}$ Future research should test the utility of proactive recruitment approaches in the Centrelink setting . This may increase retention rates and engagement with treatment in smokers recruited from Centrelink and similar social welfare agencies internationally.

A key lesson from this trial was the utility of reminding participants of follow-up interviews and reimbursement for completed interviews. The latter is consistent with the literature on the effectiveness of participant reimbursement at ensuring high retention. ${ }^{[62]}$ It was not possible to tease out which retention strategy was responsible for the high retention in our study but anecdotal comments from participants suggested that reimbursement played an important role . On balance, participant reimbursement was low cost, and potentially more resources would have been needed to attain a similar high retention rate if we had not used an incentive. It is unlikely that the study's high retention rate was the result of one single strategy e.g. reimbursement. Indeed, a multi-component approach may be the most useful way to minimise attrition in this hard-to-reach population i.e. an approach that combines a rigorous reminder and follow-up process alongside participant 
reimbursement. It is critical to have a clear plan for the retention of low-SES populations in clinical studies and understanding of key retention strategies when developing research protocols . The successful recruitment and high retention in this study can be emulated by future research teams using planned and diverse recruitment methods, rigorous participant tracking and follow-up procedures, and participant reimbursement. This requires the simultaneous use of multiple strategies including participant incentives. ${ }^{[63]}$

\section{Limitations}

IN this study low-SES was defined as being in receipt of government pension or allowance. This measure of disadvantage requires the person to qualify for means tested government income support. The sample included more heavily nicotine dependent persons because low-SES smokers who reported smoke less than 10 cigarettes per day were excluded. Those without a home or mobile telephone were excluded because the study was phone-based. Income was not included in models, because by definition our sample had a low income and $30 \%$ of the sample did not disclose their household income. Additionally we did not collect detailed data on non-consenters or those that were not eligible for study participation, with exception of reason for their non-eligibility in the CONSORT diagram. Consequently, our trial did not allow for an in-depth evaluation of reasons for non-participation among low-SES smokers, with the exception of self-reported information determined by the eligibility/consent screening as to why the participant were not eligible e.g. smoking < 10 cigarettes etc. Nonetheless, there is literature on the characteristics of smokers who do not participate in smoking cessation clinical trials. ${ }^{[64,65]}$ Finally, our study design did not allow differentiation between the individual effects of each retention strategy adopted.

\section{Conclusions}

Participant retention for clinical trials is a major challenge for intervention studies targeting lowSES populations because high participant retention is critical to the integrity of the evaluation of treatment outcomes. Overall, although our sample was constrained to low-SES smokers, the sample 
was quite diverse and encompassed a number of understudied and disadvantaged populations including: persons with mental health disorders, Indigenous Australians, those seeking drug or alcohol treatment, and persons with varying levels of general health status and mental wellbeing. Positively, however, there were no significant differences in retention rates across these population groups and overall a high retention rate was achieved. Importantly this study demonstrated useful retention strategies that achieved a more representative follow-up rate. The validity of study outcomes relates to the ability of researchers to obtain representative follow-up rates. This study adds to the understanding of factors associated with retention of low-SES smokers, and importantly with rigorous reminder and adequate participant reimbursement highlights that inequality in study retention can be overcome for sub-groups of low-SES smokers, commonly known to be underrepresented.

Future trials would benefit from following similar methods as this study, including the initiation of prompt treatment after assessing eligibility and obtaining consent. Participant reimbursement helped to achieve high retention in this study and should be more widely adopted in future studies. Future investigation of innovative ways to ensure equally high retention in low-SES participants are critical to achieving high retention rates in smoking cessation trials among low-SES smokers. Importantly future randomised trials examining retention strategies and rates should explore and disentangle the contribution of adjunct retention strategies e.g. persistent contact attempts and tracking procedures, and its yields when conducted in the absence and presence of participant incentives/reimbursement in low-SES smoking populations.

\section{What is already known on this subject?}

The inclusion of diverse population groups in research, particularly clinical trials is essential to ensure equitable benefits from research and to reduce socioeconomic disparities in health. 
There is a paucity of evidence on the factors associated with low-SES populations retention in smoking cessation studies, despite increased smoking rates and tobacco-related harms faced by these smokers.

\section{What important gaps exist in knowledge on this topic?}

A paucity of information on the most effective methods to ensure high retention in clinical trials in low-SES smokers .

\section{What this study adds?}

This study is the first to ascertain which factors were associated with retention of Australian lowSES smokers in a smoking cessation trial and to examine the association between smoking-related, health-related, behavioural, sociodemographic and recruitment source and retention.

This paper identified a retention rate of $84 \%$ at 8 -month follow-up and to our knowledge is one of the highest rates recorded in a pragmatic RCT in a low-SES smoking population.

This study highlights that rigorous reminders and participant reimbursement can prevent high attrition rates .

\section{Competing interests}

Professor Billie Bonevski has received research funding from a Pfizer Investigator Initiated Grant. Professor Robert West undertakes research and consultancy for companies that develop and manufacture smoking cessation medications. He is unpaid co-director of the National Centre for Smoking Cessation and Training, a not-for-profit organisation involved in training and assessing stop smoking practitioners.

\section{Acknowledgments}


This research is funded by a grant (APP1021862) from the Australian National Health and Medical Research Council (NHMRC). The National Drug and Alcohol Research Centre at the University of New South Wales (UNSW), Australia is supported by funding from the Australian Government under the Substance Misuse Prevention and Service Improvements Grants Fund and by infrastructure support from the UNSW, Australia. RJC is supported by a Cancer Institute New South Wales Early Career Research Fellowship (GNT14/ECF/1-46), BB is supported by a NHMRC Career Development Fellowship (GNT1063206) and RPM by a NHMRC Principal Research Fellowship (GNT1045318). GlaxoSmithKline provided NRT at a reduced cost for the study. We would like to thank Emma Black, Veronica Boland, Deborah Bradford, Danya Braunstein, Philip Clare, Jaimi Iredale, Sundresan Naicker, Joel Tibbetts, Lauren Touyz and Emily Upton for their work on the project. We acknowledge the assistance of Quitline services, and Australian Government Department of Human Services Centrelink Customer Service Centres, for assisting with recruitment and staff at the Hunter Valley Research Foundation for their diligence with data collection. 


\section{References}

1. Toerin M, Brookes ST, Metcalfe C, et al. A review of reporting of participant recruitment and retention in RCTs in six major journals. Trials 2009;10:52.

2. Lugtig P. Panel attrition: Seperating stayers, fast attriters, gradual attriters, and lurkers. Socio Meth Res 2014;43:699-733.

3. Goldberg M, Francois Chastang J, Zins M, et al. Health problems were the strongest predictors of attrition during follow-up of the GAZEL cohort. J Clin Epidemiol 2006;59:1213-21.

4. Severi E, Free C, Knight R, et al. Two controlled trials to increase participant retention in a randomized controlled trial of mobile phone-based smoking cessation support in the United Kingdom. Clin Trials 2011;8:654-60.

5. Leon AC, Demirtas H, Hedeker D. Bias reduction with an adjustment for participants' intent to drop out of a randomized controlled clinical trial. Clin Trials 2007;4:540-47.

6. Szklo M, Nieto FJ. Epidemiology, Beyond the Basics. Third Edition. . Sudbury, Ma: Jones and Bartlett; 2012.

7. Robinson KA, Dennison CR, Wayman DM, et al. Sytematic review identifies number of strategies important for retaining study participants. J Clin Epidemiol 2007;60:757-65.

8. Sprauge S, Leece P, Bhandri M, et al. Limiting loss to follow up in a multicenter randomized trial in orthopedic surgery. Contol Clin Trials 2003;24:719-25.

9. Ellenberg JH. Selection bias in observational and experimental studies. Stat Med 1994;13:557-67.

10. Hunt JR, White E. Retaining and tracking cohort study members. Epidemiol Rev 1998;20:57-70.

11. Shih WJ. Problems in dealing with missing data and informative censoring in clinical trials. Curr Control Trials in Cardiovasc Med 2002;3:4.

12. Twisk J, de Vente W. Attrition in longitudinal studies. How to deal with missing data. J Clin Epidemiol 2002;55:329-37. 
13. Leak C, Goggins K, Schildcrout JS, et al. Effect of health literacy on research follow-up. $J$ Health Commun 2015;20:83-91.

14. Woolard RH, Carty K, Wirtz P, et al. Research fundamentals: Follow-up of subjects in clinical trials: Addressing subject attrition. Acad Emerg Med 2004;11:859-66.

15. Bonevski B, Randell M, Paul C, et al. Reaching the hard-to-reach: a systematic review of strategies for improving health and medical research with socially disadvantaged groups. BMC Med Res Methodol 2014;14:42.

16. Blumental DS, Sung J, Coates R, et al. Recruitment and retention of subjects for a longitudinal cancer prevention study in an inner-city black community. Health Serv Res 1995;30:197-205.

17. Cunningham R, Walton MA, Tripathi SP, et al. Tracking inner city subtance users from the emergency department: how many contacts does it take? . Acad Emerg Med 2008;15:136-43.

18. Ramos-Gomez F, Chung LH, Gonzalez Beristain R, et al. Recruiting and retaining pregnant women from a community health center at the US-Mexico border for the Mothers and Youth Access clinical trial. ClinTrials 2008;5:336-46.

19. Brueton VC, Tierney JF, Stenning S, et al. Strategies to improve retention in randomised trials: a Cochrane systematic review and meta-analysis. BMJ Open 2013;4:e003821.

20. Ngo-Metzger Q, Kaplan SH, Sorkin DH, et al. Surveying minorities with limited-English proficiency: does data collection method affect data quality among Asian Americans? Med Care 2004;42:893-900.

21. El-Khorazaty NM, Johnson AA, Kiely M, et al. Recruitment and retention of low-income minority women in a behavioural intervention to reduce smoking, depression, and intimate partner violence during pregnancy. BMC Public Health 2007;7:233.

22. Faseru B, Cox LS, Bronars CA, et al. Design recruitment and retention of African-American smokers in a pharmacokinetic study. BMC Med Res Methodol 2010;10:6. 
23. Woods MN, Harris KJ, Mayo MS, et al. Participation of African Americans in a smoking cessation trial: a quantitiative and qualititative evaluation study. J Natl Med Assoc 2002;94:609-18.

24. Borrelli B, Hogan JW, Bock B, et al. Predictors of quitting and dropout among women in a clinic-based smoking cessation program. Psychol of Addict Behav 2002;16:22-27.

25. Leeman RF, Quiles ZN, Molinelli LA, et al. Attrition in a multi-component smoking cessation study for females. Tob Induc Dis 2006;3:59-71.

26. Nevid JS, Javier RA, Moulton JL. Factors predicting participant attrition in a communitybased, culturally specific smoking cessation program for Hispanic smokers. Health Psychol 1996;15:226-29.

27. Bowen DJ, McTiernan A, Powers D, et al. Recruiting women into a smoking cessation program: Who might quit? Women Health 2000;31:41-58.

28. Curtin L, Brown RA, Sales SD. Determinants of attrition from cessation treatment in smokers with a history of major depressive disorder. Psychol Addict Behav 2000;14:134-42.

29. Fortman SP, Killen JD. Who shall quit? Comparison of volunteer and population-based recruitment in two minimal-contact smoking cessation studies. Am J Epidemiol 1994;140:39-51.

30. Greenberger P, Knab S. Society workshop investigates barriers to recruitment and retention of women in clinical research. J Womens Health Gend Based Med 2000;9:817-18.

31. Beaver KM. Intelligence and selective attrition in a nationally representative and longitudinal sample of Americans. Pers Indiv Dif 2013;55:157-61.

32. Lynham D, Moffitt T, Stouthamer-Loeber M. Explaining the relation between IQ and deliniquency: Class, race, test motivation, school failure, or self control? . J Abnorm Psychol 1993;102:187-96.

33. de Graaf R, Bijl RV, Smit F, et al. Psychiatric and sociodemographic predictors of attrition in a longitudinal study: The Netherlands Mental Health Survey and Incidence Study (NEMESIS). Am J Epidemiol 2000;152:1039-47. 
34. Deeg DJ, van Tilburg T, Smit JH, et al. Attrition in the Longitudinal Aging Study Amsterdam. The effect of differential inclusion in side studies. . J Clin Epidemiol 2002;55:319-28.

35. Morrison TC, Wahlgreen DR, Hovell MF, et al. Tracking and follow-up of 19,915 adolescents: minimizing attrition bias. Control Clin Trials 1997;18:383-96.

36. Siddiqui O, Flay BR, Hu FB. Factors affecting attrition in a longitudinal smoking prevention study. Prev Med 1996;25:554-60.

37. Nowak C, Sharif F, Eischen S, et al. Retention of homeless smokers in the Power to Quit study. Nicotine Tob Res 2014; In press.

38. Courtney RJ, Bradford D, Martire KA, et al. A randomized clinical trial of a financial education intervention with nicotine replacement therapy (NRT) for low socio-economic status Australian smokers: a study protocol. Addiction 2014;109:1602-11.

39. Borland R, Yong HH, O'Connor RJ, et al. The reliability and predictive validity of the Heaviness of Smoking Index and its two components: findings from the International Tobacco Control Four Country study. Nicotine Tob Res 2010;12 Suppl:S45-50.

40. Babor TF, Higgins-Biddle JC, Saunders JB, et al. AUDIT : the Alcohol Use Disorders Identification Test : Guidelines for use in primary health care. Geneva: World Health Organization, 2001.

41. Gebauer L, LaBrie R, Shaffer HJ. Optimizing DSM-IV-TR classification accuracy: a brief biosocial screen for detecting current gambling disorders among gamblers in the general household population. Can J Psychiatry 2010;55:82-90.

42. Lovibond SH, Lovibond PF. Manual for the Depression Anxiety Stress Scales. 2nd ed. Sydney: Psychology Foundation; 1995.

43. EuroQol Group. EuroQol--a new facility for the measurement of health-related quality of life. Health Policy 1990;16:199-208.

44. Travers P, Robertson F. Deprivation Standards Project. The Flinders University of South Australia, Report prepared for the Department of Social Security., 1995. 
45. Siahpush M, Carlin JB. Financial stress, smoking cessation and relapse: Results from a prospective study of an Australian national sample. Addiction 2006;101:121-27.

46. Australian Bureau of Statistics. Household Expenditure Survey, Australia, user guide, 19981999. Canberra: AGPS, 2000.

47. Brownlee H. Measuring living standards, AIFS Australian living standards study, Paper No.

1. Melbourne: Australian Institute of Family Studies, 1990.

48. NCS Pearson Corporation. Test of Premorbid Functioning. Oxford, UK: NCS Pearson Corporation.: 2011.

49. Wechsler D. Wechsler Adult Intelligence Scale - Fourth Edition (WAIS-IV) Australian and New Zealand Language Adaptation. Sydney: Pearson Clinical and Talent Management: 2008.

50. Spinella M. Normative data and a short form of the Barratt Impulsiveness Scale. Int J Neurosci 2007;117:359-68.

51. Australian Bureau of Statistics. Socio-Economic Indexes for Areas (SEIFA) - Technical Paper 2011. Canberra: ABS; 2011.

52. StataCorp. Stata Statistical Software: Release 13. College Station, TX: StataCorp LP; 2013.

53. Baker A, Richmond R, Haile M, et al. A randomized controlled trial of a smoking cessation intervention among people with psychotic disorder. Am J Psychiatry 2006;163:1934-42.

54. Courtney RJ, Naicker S, Shakeshaft A, et al. Smoking cessation among low-socioeconomic status and disadvantaged population groups: A systematic review of research output. Int J Environ Res Public Health 2015;12:6403-22.

55. Bryant J, Bonevski B, Paul C, et al. A systematic review and meta-analysis of the effectiveness of behavioural smoking cessation interventions in selected disadvantaged groups. Addiction 2011;106:1568-85.

56. Baker A, Richmond R, Haile M, et al. Characteristics of smokers with a psychotic disorder and implications for smoking interventions. Psychiatry Res 2007;150:141-52. 
57. Lee S, Hayes RB, McQuaid EL, et al. Predictors of retention in smoking cessation treatment among Latino smokers in the Northeat United States. Health Educ Res 2010;25:687-97.

58. Shelley D, Cantrell J, Wong S, et al. Smoking cessation among sheltered homeless: A pilot. Am J Health Behav 2010;34:544-52.

59. Kim R, Kickman N, Gali K, et al. Maximising retention with high risk participants in a clinical trial. Am J Health Prom 2014;28:268-74.

60. Mapstone J, Elbourne D, Roberts I. Strategies to improve recruitment to research studies. Cochrane Database Syst Rev 2007;18:MR000013.

61. Collins BN, Wileyto EP, Hovell MF, et al. Proactive recruitment predicts participant retention to end of treatment in a secondhand smoke reduction trial with low-income maternal smokers. Transl Behav Med 2011;1:394-99.

62. Festinger DS, Marlowe DB, Dugosh KL, et al. Higher magnitude cash payments improve research follow-up rates without increasing drug use or perceived coercion. Drug Alcohol Depend 2008;96:128-35.

63. Nicholson LM, Schwirian PM, Klein EG, et al. Recruitment and retention stratagies in longitudinal clinical studies with low-income populations. Contemp Clin Trials 2011;32:353-62.

64. Boneski B, Twyman L, Paul C, et al. Comparing socially disadvantaged smokers who agree and decline to participate in a randomised smoking cessation trial. BMJ Open 2015;5:e008419. 65. Graham AL, Papandonatos GD, DePue JD, et al. Lifetimes characteristics of participants and non-participants in a smoking cessation trial: Implications for external validity and public health impact. Ann Behav Med 2008;35:295-307. 